\title{
Who is the Key Player?
}

\section{A Network Analysis of Juvenile Delinquency ${ }^{1}$}

\author{
Lung-Fei Lee ${ }^{2} \quad$ Xiaodong Liu $^{3} \quad$ Eleonora Patacchini ${ }^{4}$ \\ Yves Zenou ${ }^{5}$
}

February, 2020

\footnotetext{
${ }^{1} \mathrm{~A}$ previous version of this paper was circulated under the title: "Criminal Networks: Who is the Key Player?". We thank the editor, Christian Hansen, an associate editor as well as two anonymous referees for very helpful comments. This research uses data from Add Health, a program project designed by J. Richard Udry, Peter S. Bearman, and Kathleen Mullan Harris, and funded by a grant P01-HD31921 from the National Institute of Child Health and Human Development, with cooperative funding from 17 other agencies. Special acknowledgment is due Ronald R. Rindfuss and Barbara Entwisle for assistance in the original design. Persons interested in obtaining data files from Add Health should contact Add Health, Carolina Population Center, 123 W. Franklin Street, Chapel Hill, NC 275162524 (addhealth@unc.edu). No direct support was received from grant P01-HD31921 for this analysis.

${ }^{2}$ The Ohio State University. E-mail: lee.1777@osu.edu.

${ }^{3}$ Corresponding author. University of Colorado Boulder. E-mail: xiaodong.liu@colorado.edu.

${ }^{4}$ Cornell University, EIEF and CEPR. E-mail: ep454@cornell.edu.

${ }^{5}$ Monash University, IFN and CEPR. E-mail: yves.zenou@monash.edu.
} 


\begin{abstract}
This paper presents a methodology for empirically identifying the key player, whose removal from the network leads to the optimal change in aggregate activity level in equilibrium (Ballester et al., 2006), allowing the network links to rewire after the removal of the key player. First, we propose an IV-based estimation strategy for the social-interaction effect, which is needed to determine the equilibrium activity level of a network, taking into account the potential network endogeneity. Next, to simulate the network evolution process after the removal of the key player, we adopt the general network formation model in Mele (2017) and extend it to incorporate the unobserved individual heterogeneity in link formation decisions. We illustrate the methodology by providing the key player rankings in juvenile delinquency using unique information on friendship networks among U.S. teenagers. We find that the key player is not necessarily the most active delinquent or the delinquent who is the highest in standard (not microfounded) centrality measures. We also find that, compared to a policy that removes the most active delinquent from the network, a key-player-targeted policy leads to a much higher delinquency reduction.
\end{abstract}

Key words: linear social interaction models, network centrality measures, network endogeneity, network formation, key player policies.

JEL Classification: A14, C31, D85, K42, Z13 


\section{Introduction}

The increasing availability of social network data, computational power and statistical methods has stimulated a growing literature in different disciplines about social network effects. A crucial aspect of social network analysis is the identification of the key player, who hold a vital position in a network regarding to some criteria. The majority of the existing empirical studies adopts a definition of the key player based on the individual prominence in terms of network centrality measures, e.g., the most connected individual or the individual with the highest eigenvector centrality (for a review, see Zenou, 2016).

Ballester et al. (2006) present a theory that characterizes the key player as the agent who, once removed from the network, leads to the optimal change in aggregate activity level in equilibrium. This theory takes into account both the network topology and the intensity of social interactions in determining the identity of the key player.

As the effect of a policy diffuses in a network through social interactions, targeting the key player would significantly improve the effectiveness of policy interventions.

While this idea is appealing in theory, its empirical implementation is challenging for two main reasons. First, it requires the estimation of the social-interaction effect, that is the impact of an agent's peers' behavior on her own behavior, which is a well-known difficult task (e.g., de Paula, 2017). The difficulty is even greater when there exists unobserved factors driving both individual behavior and the underlying network formation process, so that the social network is endogenous. To estimate the social-interaction effect, the conventional estimation strategy (e.g., Bramoullé et al., 2009; Calvó-Armengol et al., 2009; Liu and Lee, 2010) uses the exogenous characteristics of the friends' friends as instrumental variables (IVs) for the behavior of the friends. However, when the social network is endogenous, this IV strategy is 
clearly invalid because who the friends are becomes endogenous.

Second, it is debatable what the counterfactual should be after the key player is hypothetically removed from the network. Under the assumption that the rest of the network remains the same after the removal of a node (henceforth referred to as the invariant network assumption), Ballester et al. (2006) and Ballester and Zenou (2014) have shown that the key player is the agent with the highest (generalized) intercentrality measure. The invariant network assumption is reasonable if the key player analysis is considered as a short-run policy analysis, since it is unlikely for the remaining agents to form new links in the short period of time after the removal of the key player. In the long run, the invariant network assumption can be justified if network links are pairwise independent since, in this case, the removal of a node and its associated links would not affect the other links in the network. If the pairwise independence assumption for network links does not hold, then one needs to estimate a network formation model to produce meaningful counterfactuals for the long-run key player analysis.

In this paper, we present a methodology to empirically implement the key player analysis that tackles the challenges described above. We adopt the general network formation model in Mele (2017) and extend it to incorporate the unobserved individual heterogeneity in link formation decisions. Combining the network formation model and the social interaction model, we are able to explicitly model the origin of the network endogeneity as the correlation of unobserved individual heterogeneities in these two models. Under this setup, we propose an IV-based estimation strategy to estimate the social-interaction effect. To overcome the problem of network endogeneity, we first run a simple logistic regression to obtain predicted probabilities of forming links between every pair of agents in the network based on exogenous dyadic characteristics (i.e., whether the two agents are of the same age, gender, race, etc.). 
Then, we use the average exogenous characteristics of an agent's peers, weighted by the predicted probabilities of forming links, (or, roughly speaking, the exogenous characteristics of the predicted friends) as IVs for the behavior of the agent's friends. By design, the IVs are valid even if the social network is endogenous. This IV-based estimation strategy does not require the estimation of the full network formation model and, thus, is computationally simple to implement. Furthermore, to alleviate the potential weak IV problem when the exogenous dyadic characteristics are not very informative in explaining the formation of network links, we introduce an additional quadratic moment condition based on the correlation structure of the error term and propose a generalized method of moments (GMM) estimator that utilizes both linear and quadratic moment conditions to improve estimation efficiency. Finally, to conduct the key player analysis, we estimate the general network formation model with unobserved individual heterogeneities using the Bayesian double MetropolisHastings (DMH) algorithm (Liang, 2010; Mele, 2017). The unobserved individual heterogeneities are treated as unknown parameters and sampled together with other model parameters from the joint posterior distribution following the Bayesian data augmentation approach. With the estimated network formation model, we can simulate the network evolution process after an agent is removed from the network, and conduct the key player analysis without imposing the restrictive invariant network assumption. As a by-product, our framework allows us to test for the pairwise independence of network links.

To illustrate our methodology, we provide the key-player rankings in juvenile delinquency using data from the National Longitudinal Study of Adolescent Health (Add Health). We find that the key player is not necessarily the most active delinquent in the network. We also find that it is not straightforward to determine which delinquent should be removed from a network based on other standard network centrality 
measures (which are not microfounded). Finally, we find that, compared to a policy that removes the most active delinquent from the network, the key-player-targeted policy is more effective in reducing the aggregate delinquent activity level as it takes full advantage of the social interaction effect.

The rest of the paper unfolds as follows. In Section 2, we introduce the network game, characterize the equilibrium, and define the key player. Our estimation strategy is described in Section 3. Section 4 presents the empirical results. In Section 5, we conduct the key player analysis. Finally, Section 6 concludes.

\section{Microfoundation}

\subsection{Network and utility}

Consider a finite set of agents $\mathcal{N}=\{1, \ldots, n\}$ with each agent corresponding to a node in a network $g$. We keep track of social connections in the network through an $n \times n$ zero-diagonal adjacency matrix $G=\left[g_{i j}\right]$, where $g_{i j}=1$ if agent $i$ forms a link to agent $j$ and $g_{i j}=0$ otherwise.

Given the network structure, agents choose how much effort to exert in delinquent activities. We denote by $y_{i}$ the effort level of agent $i$ and by $y=\left(y_{1}, \ldots, y_{n}\right)^{\prime}$ the population effort profile in the network. Each agent $i$ selects an effort $y_{i} \geq 0$ and obtains a utility $\mathcal{U}_{i}(y)$ given by

$$
\mathcal{U}_{i}(y)=\underbrace{\left(\pi_{i}+\lambda \sum_{j=1}^{n} g_{i j} y_{j}\right) y_{i}}_{\text {payoff }}-\underbrace{\left(\varsigma y_{i}+\frac{1}{2} y_{i}^{2}\right)}_{\text {cost }} .
$$

This utility has a standard cost-payoff structure as in Becker (1968). The payoff increases in own effort $y_{i}$ with the marginal payoff given by $\pi_{i}+\lambda \sum_{j=1}^{n} g_{i j} y_{j}$. The term $\pi_{i}$ reflects individual heterogeneity of "productivity" in delinquent activities, 
and is given by

$$
\pi_{i}=\alpha+X_{i} \beta_{1}+\bar{X}_{i} \beta_{2}+u_{i}
$$

where $X_{i}$ is a row vector of observable exogenous characteristics of agent $i$ (e.g. age, sex, race, parental education, etc.), $\bar{X}_{i}=\sum_{j=1}^{n} g_{i j} X_{j} / \sum_{j=1}^{n} g_{i j}$ is the average exogenous characteristics of agent $i$ 's connections, with the coefficient vector $\beta_{2}$ capturing contextual effects (Manski, 1993), and $u_{i}$ representing the unobservable (to the econometrician) characteristics of agent $i$.

Compared to the standard crime model (Becker, 1968), the marginal payoff in the utility function has a new component $\lambda \sum_{j=1}^{n} g_{i j} y_{j}$, which reflects the impact of the total effort of an agent's connections on her own "productivity". Indeed, an agent may benefit directly from the effort of her connections if they are co-offenders in some delinquent activity. An agent may also benefit indirectly through the form of knowhow sharing about delinquent behavior with her friends. We assume $\lambda \geq 0$, which implies the more delinquent connections an agent has and the more these connections are involved in delinquent activities, the higher is the marginal payoff of the agent's own delinquent effort. We call $\lambda$ the social-interaction coefficient.

The cost part of the utility function (1) has two components. The cost of being caught, given by $\varsigma y_{i}$, is assumed to increase with the effort level $y_{i}$, as the severity of the punishment increases with one's involvement in delinquent activities. Also, individuals have a direct cost of exerting effort given by $\frac{1}{2} y_{i}^{2}$.

\subsection{Equilibrium and key player}

In equilibrium, each agent maximizes her utility and the best-response function is given by:

$$
y_{i}=\lambda \sum_{j=1}^{n} g_{i j} y_{j}+\beta_{0}+X_{i} \beta_{1}+\bar{X}_{i} \beta_{2}+u_{i}
$$


where $\beta_{0}=\alpha-\varsigma$. Let $\rho(A)$ denote the spectral radius of a square matrix $A$, and $I_{n}$ denote the $n \times n$ identity matrix. If $|\lambda|<1 / \rho(G)$, then $I_{n}-\lambda G$ is nonsingular and the network game has a unique interior Nash equilibrium in pure strategies with the equilibrium effort vector $y^{*}=\left(y_{1}^{*}, \ldots, y_{n}^{*}\right)^{\prime}$ given by:

$$
y^{*} \equiv y^{*}(g)=\left(I_{n}-\lambda G\right)^{-1}\left(\beta_{0} \iota_{n}+X \beta_{1}+\bar{X} \beta_{2}+u\right),
$$

where $\iota_{n}$ is an $n \times 1$ vector of ones, $X=\left(X_{1}^{\prime}, \cdots, X_{n}^{\prime}\right)^{\prime}, \bar{X}=\left(\bar{X}_{1}^{\prime}, \cdots, \bar{X}_{n}^{\prime}\right)^{\prime}$, and $u=\left(u_{1}, \cdots, u_{n}\right)^{\prime}$.

The key player in a network is the agent whose removal from the network leads to the largest reduction in the total equilibrium effort level (Ballester et al., 2006). More formally, let $g_{-i}$ denote the network obtained by removing the $i$ th node from network $g$, and $\|\cdot\|_{1}$ denote the $L_{1}$ matrix norm. Then the key player is defined as

$$
i^{*}=\arg \max _{i \in \mathcal{N}}\left(\left\|y^{*}(g)\right\|_{1}-\left\|y^{*}\left(g_{-i}\right)\right\|_{1}\right)=\arg \min _{i \in \mathcal{N}}\left\|y^{*}\left(g_{-i}\right)\right\|_{1},
$$

where $y^{*}(g)$ and $y^{*}\left(g_{-i}\right)$ are equilibrium effort vectors for the underlying networks $g$ and $g_{-i}$, respectively, defined in Equation (4).

Let $[A]_{i j}$ denote the $(i, j)$ th entry of a matrix $A$ and $[b]_{i}$ denote the $i$ th entry of a vector $b$. Under the invariant network assumption, Ballester et al. (2006) and Ballester and Zenou (2014) show that, in the case without contextual effects (i.e., $\beta_{2}=0$ ), the key player is the agent with the highest intercentrality measure given by

$$
\frac{\left[\left(I_{n}-\lambda G\right)^{-1} \pi\right]_{i} \sum_{j=1}^{n}\left[\left(I_{n}-\lambda G\right)^{-1}\right]_{j i}}{\left[\left(I_{n}-\lambda G\right)^{-1}\right]_{i i}},
$$

where $\pi$ denotes the vector of individual heterogeneity terms defined in Equation (2); and, in the case with contextual effects (i.e., $\beta_{2} \neq 0$ ), the key player is the agent with 
the highest generalized intercentrality measure given by

$$
\underbrace{\left\|\left(I_{n}-\lambda G\right)^{-1}\left(\pi-\pi_{[i]}\right)\right\|_{1}}_{\text {contextual variable change effect }}+\underbrace{\frac{\left[\left(I_{n}-\lambda G\right)^{-1} \pi_{[i]}\right]_{i} \sum_{j=1}^{n}\left[\left(I_{n}-\lambda G\right)^{-1}\right]_{j i}}{\left[\left(I_{n}-\lambda G\right)^{-1}\right]_{i i}}}_{\text {network structure change effect }},
$$

where $\pi_{[i]}$ is calculated based on the network consisting $g_{-i}$ and the isolated node $i$. The generalized intercentrality highlights the fact that when an agent is removed from a network, two effects are at work. The first one is the contextual variable change effect, which is due to the change in $\pi$ after the removal of an agent. The second effect is the network structure change effect, which captures the change in the adjacency matrix when an agent is removed. More generally, the generalized intercentrality measure accounts for both one's exposure to the rest of the group and one's contribution to every other exposure.

The invariant network assumption makes sense in the short run, since it is unlikely for the remaining agents to form new links in the short period of time after the removal of the key player. In the long run, the invariant network assumption can be justified if network links are pairwise independent. In this paper, we consider a general network formation model that allows us to test the pairwise independence assumption of network links. Furthermore, we propose an estimation strategy for Equation (3) taking into account the endogeneity of network links, and demonstrate how to empirically identify the key player with and without the invariant network assumption. 


\section{2SLS and GMM Estimation with Endogenous Networks}

\subsection{Econometric model}

In matrix form, the best-response function given by Equation (3) can be written as

$$
y=\lambda G y+\beta_{0} \iota_{n}+X \beta_{1}+\bar{X} \beta_{2}+u,
$$

where the error term $u$ is assumed to be independently distributed but allowed to be heteroskedastic with $\Sigma \equiv \mathrm{E}\left(u u^{\prime}\right)=\operatorname{diag}\left\{\sigma_{i}^{2}\right\}$ being a diagonal matrix. To model the potential endogeneity of the adjacency matrix $G$, we follow Mele (2017) and assume the corresponding network $g$ is realized with the probability

$$
\operatorname{Pr}(g)=\frac{\exp [\mathcal{Q}(g)]}{\sum_{\widetilde{g} \in \mathcal{G}_{n}} \exp [\mathcal{Q}(\widetilde{g})]},
$$

where $\mathcal{G}_{n}$ is the set of all networks with $n$ nodes and $\mathcal{Q}(g)$ is the potential function (Monderer and Shapley, 1996). The potential function aggregates individual incentives to change the status-quo network and takes the form

$$
\mathcal{Q}(g)=\sum_{i=1}^{n} \sum_{j=1}^{n} g_{i j}\left[\delta_{0}+W_{i j} \delta_{1}+m_{i j}(g)+\eta_{i}+\eta_{j}\right]
$$

where $W_{i j}$ is a row vector of homophily measures reflecting the similarity in exogenous characteristics between agents $i$ and $j, m_{i j}(g)$ collects terms that depend on other links in the network (e.g., the number of common friends of agents $i$ and $j$ given by $\left.\sum_{k \neq i, j} g_{j k} g_{k i}\right)$, and $\eta_{i}$ represents the unobserved individual heterogeneity. Our estimation strategy does not rely on the parametric specification of $m_{i j}(g)$. The specification of $m_{i j}(g)$ and more detailed discussion of the potential function can be

found in Section 5. In this setup, the endogeneity of $G$ is captured by the correlation 
between the unobserved individual heterogeneity terms $u_{i}$ and $\eta_{i}$ in delinquent activities and link formation decisions respectively. Our goal is to consistently estimate the parameters $\theta=\left(\lambda, \beta_{0}, \beta_{1}^{\prime}, \beta_{2}^{\prime}\right)^{\prime}$ in Equation (6) taking into account this endogeneity problem.

\subsection{Linear moment condition and 2SLS}

When the adjacency matrix $G$ is exogenous, linear social interaction models in the form of Equation (6) can be estimated by the 2SLS method based on the linear moment condition $Z^{\prime} u(\theta)=0$, where $Z$ is a matrix of IVs consisting of linearly independent columns of $\left[\iota_{n}, X, \bar{X}, G \iota_{n}, G X, G \bar{X}\right]$ and

$$
u(\theta)=y-\lambda G y-\beta_{0} \iota_{n}-X \beta_{1}-\bar{X} \beta_{2} .
$$

The parameters in Equation (6) can be identified, provided that a certain level of intransitivity exists in the network such that the usual rank condition of IV estimators holds (Bramoullé et al., 2009; Liu and Lee, 2010).

However, if the unobserved individual heterogeneity terms $u_{i}$ and $\eta_{i}$ in Equations (6) and (8) are correlated, then $G$ is endogenous and the IV matrix $Z$ is no longer valid. In this case, the 2SLS method can be remedied by replacing the observed adjacency matrix $G$ in the IV matrix by a predicted adjacency matrix $\widehat{G}$ based on exogenous covariates (Kelejian and Piras, 2014). To that end, we need to remove $\eta_{i}, \eta_{j}$ and $m_{i j}(g)$ from Equation (8) as these terms are correlated with the error term in Equation (6). The substitution of the remainder of Equation (8) into Equation (7) yields

$$
\operatorname{Pr}(g)=\frac{\exp \left[\sum_{i=1}^{n} \sum_{j=1}^{n} g_{i j}\left(\delta_{0}+W_{i j} \delta_{1}\right)\right]}{\sum_{\widetilde{g} \in \mathcal{G}_{n}} \exp \left[\sum_{i=1}^{n} \sum_{j=1}^{n} \widetilde{g}_{i j}\left(\delta_{0}+W_{i j} \delta_{1}\right)\right]}=\prod_{i=1}^{n} \prod_{j=1}^{n} \frac{\exp \left[g_{i j}\left(\delta_{0}+W_{i j} \delta_{1}\right)\right]}{1+\exp \left(\delta_{0}+W_{i j} \delta_{1}\right)},
$$


which implies links are pairwise independent and each link is formed with probability

$$
\operatorname{Pr}\left(g_{i j}=1\right)=\frac{\exp \left(\delta_{0}+W_{i j} \delta_{1}\right)}{1+\exp \left(\delta_{0}+W_{i j} \delta_{1}\right)}
$$

Based on Equation (9), we define

$$
\widehat{g}_{i j}=\frac{\exp \left(\widehat{\delta}_{0}+W_{i j} \widehat{\delta}_{1}\right)}{1+\exp \left(\widehat{\delta}_{0}+W_{i j} \widehat{\delta}_{1}\right)}
$$

where $\widehat{\delta}_{0}$ and $\widehat{\delta}_{1}$ are obtained from a logistic regression of $g_{i j}$ on $W_{i j}$. To make sure the predicted adjacency matrix is uniformly bounded in row and column sums, we normalize $\widehat{g}_{i j}$ by dividing it by $\widehat{d}=\max \left\{\max _{i} \sum_{j=1}^{n} \widehat{g}_{i j}, \max _{j} \sum_{i=1}^{n} \widehat{g}_{i j}\right\}$ (Kelejian and Prucha, 2010), and define the $(i, j)$ th element of the predicted adjacency matrix $\widehat{G}$ as $\widehat{g}_{i j} / \widehat{d}$ if $i \neq j$ and zero otherwise.

The IV matrix based on the predicted adjacency matrix $\widehat{G}$ is denoted by $\widehat{Z}$ and includes linearly independent columns of $\left[\iota_{n}, X, \widehat{\bar{X}}, \widehat{G} \iota_{n}, \widehat{G} X, \widehat{G} \widehat{\bar{X}}\right]$, where $\widehat{\bar{X}}=$ $\left(\widehat{\bar{X}}_{1}^{\prime}, \cdots, \widehat{\bar{X}}_{n}^{\prime}\right)^{\prime}$ with $\widehat{\bar{X}}_{i}=\sum_{j=1}^{n} \widehat{g}_{i j} X_{j} / \sum_{j=1}^{n} \widehat{g}_{i j}$. The corresponding 2SLS estimator is given by

$$
\widehat{\theta}_{2 s l s}=\left[\underline{X}^{\prime} \widehat{Z}\left(\widehat{Z}^{\prime} \widehat{Z}\right)^{-1} \widehat{Z}^{\prime} \underline{X}\right]^{-1} \underline{X}^{\prime} \widehat{Z}\left(\widehat{Z}^{\prime} \widehat{Z}\right)^{-1} \widehat{Z}^{\prime} y
$$

where $\underline{X}=\left[G y, \iota_{n}, X, \bar{X}\right]$. The consistency of the proposed 2SLS estimator does not rely on the consistency of the estimator $\widehat{\delta}=\left(\widehat{\delta}_{0}, \widehat{\delta}_{1}^{\prime}\right)^{\prime}$. Suppose $\widehat{\delta}$ converges in probability to a well defined limit $\delta^{*}$ (not necessarily the true parameter value) such that $\sqrt{n}\left(\widehat{\delta}-\delta^{*}\right)=O_{p}(1)$. Let $G^{*}$ and $\bar{X}^{*}$ be defined in the same way as $\widehat{G}$ and $\widehat{\bar{X}}$ respectively, with $\widehat{g}_{i j}$ in $\widehat{G}$ and $\widehat{\bar{X}}$ replaced by

$$
g_{i j}^{*}=\frac{\exp \left(\delta_{0}^{*}+W_{i j} \delta_{1}^{*}\right)}{1+\exp \left(\delta_{0}^{*}+W_{i j} \delta_{1}^{*}\right)} .
$$


Then, the parameters in Equation (6) can be identified via the IV matrix $Z^{*}$ consisting of linearly independent columns of $\left[\iota_{n}, X, \bar{X}^{*}, G^{*} \iota_{n}, G^{*} X, G^{*} \bar{X}^{*}\right]$, as long as $X_{i}$ and $W_{i j}$ are exogenous and the usual rank condition of IV estimators holds (Kelejian and Prucha, 1998; Lee, 2007). Under some regularity assumptions, we can show that $\widehat{Z}^{\prime} u(\theta)=0$ is asymptotically equivalent to the infeasible linear moment condition $Z^{* \prime} u(\theta)=0$, and hence $\widehat{\theta}_{2 s l s}$ defined in Equation (10) is root- $n$ consistent and asymptotically normal. The asymptotic distribution of $\widehat{\theta}_{2 s l s}$ is given in Appendix A.

\subsection{Quadratic moment condition and GMM}

The IVs based on the predicted adjacency matrices are likely to be weak if the dyadic characteristics $W_{i j}$ are not very informative in explaining the formation of network links. To alleviate the potential weak IV problem, we introduce a quadratic moment condition for the estimation of Equation (6).

We maintain the same assumptions and notations as in the previous subsection. As $G^{*}$ is exogenous with a zero diagonal, $\mathrm{E}\left(u^{\prime} G^{*} u\right)=\operatorname{tr}\left(G^{*} \Sigma\right)=\operatorname{tr}\left(G^{*} \operatorname{diag}\left\{\sigma_{i}^{2}\right\}\right)=0$, which suggests an infeasible quadratic moment condition $u(\theta)^{\prime} G^{*} u(\theta)=0$. Under some regularity assumptions, we can show that the feasible quadratic moment condition $u(\theta)^{\prime} \widehat{G} u(\theta)=0$ is asymptotically equivalent to $u(\theta)^{\prime} G^{*} u(\theta)=0$.

Combining the linear and quadratic moment conditions, the GMM estimator is given by

$$
\widehat{\theta}_{g m m}=\arg \min h(\theta)^{\prime} \widehat{\Omega}^{-1} h(\theta),
$$

where $h(\theta)=\left[u(\theta)^{\prime} \widehat{Z}, u(\theta)^{\prime} \widehat{G} u(\theta)\right]^{\prime}$ and $n^{-1} \widehat{\Omega}$ is a consistent estimator of the variancecovariance matrix of the moment function $h(\theta)$. As should be obvious following the above discussion, the consistency of the proposed GMM estimator does not rely on the

consistency of the estimator $\widehat{\delta}$. Indeed, it is sufficient that $\widehat{\delta}$ converges in probability to a well defined limit $\delta^{*}$ such that $\sqrt{n}\left(\widehat{\delta}-\delta^{*}\right)=O_{p}(1)$. This would allow us to 
apply the results in the spatial econometrics literature (Kelejian and Prucha, 2010; Lin and Lee, 2010) to establish the root- $n$ consistency and asymptotic normality of the proposed GMM estimator. The asymptotic distribution of the proposed GMM estimator is given in Appendix A. In Appendix A, we also conduct some Monte Carlo simulations to investigate the finite sample performance of the proposed 2SLS and GMM estimators.

\section{Empirical Illustration}

\subsection{Data}

To demonstrate feasibility of the proposed method, we provide an illustrative example using data from the Add Health survey. The survey collected data on the social environment of students in grades 7-12 from roughly 130 private and public schools in the Unites States in the academic year 1994-95. Every student attending the sampled schools on the interview day completed a questionnaire (in-school survey) asking questions on basic socio-demographic characteristics. In addition, students were asked to identify their friends (up to five males and five females) from the school roster. A subset of students selected from the rosters of the sampled schools, was then asked to complete a longer questionnaire containing more sensitive individual and household information (in-home survey).

To alleviate concerns of network misspecification and missing values in variables of interest, we focus our analysis on schools where (i) more than $90 \%$ of the students participated the in-school survey and all friendship nominations of the students taking the in-school survey can be tracked, (ii) every student taking the in-school survey was also interviewed at home (where parental information is properly recorded), and (iii) less than $10 \%$ of the students have missing values in the variables of interest. One school (school \#7) satisfies these criteria. This is a midsize school with 181 students, 
where $100 \%$ of its students participated both the in-school and in-home surveys and all friendship nominations are properly recorded. In this school, there are only 8 students with missing values in variables of interest (dropped from the sample). In our final sample of 173 students, except for 4 isolated students (i.e., students who did not nominate any friends and were not nominated by anyone as a friend), all students are directly or indirectly connected in a friendship nomination network as shown in Figure 1, where $g_{i j}=1$ if student $i$ nominated student $j$ as a friend in the in-school survey. On average, the students in our sample nominated 5.57 friends, with a standard deviation of 2.82 .

\section{[Insert Figure 1 here]}

The dependent variable of our analysis is constructed based on how often a student participated in activities measuring the propensity to engage in delinquent behavior, including smoking cigarettes, drinking alcohol, getting drunk, racing on a bike or in a car, being in danger due to dare, lying to parents, and skipping school, during the last 12 months. A student's participation frequency in each of these activities is coded using an ordinal scale ranging from 0 (i.e. never participate) to 6 (i.e. nearly everyday). The dependent variable is the average participation frequency in these activities. In the sample considered, only 11 students reported never participating in any of the listed delinquent activities during the last 12 months and, thus, have a value of zero in the dependent variable. The list of variables used in the empirical analysis, with their definition and summary statistics, can be found in Table 1.

\section{[Insert Table 1 here]}

\subsection{Estimation results}

We consider the following estimators for Equation (6), namely, (a) "2SLS-1", the conventional 2SLS estimator with the IV matrix $Z=\left[\iota_{n}, X, \bar{X}, G \iota_{n}, G X, G \bar{X}\right]$; (b) 
"2SLS-2", the 2SLS estimator defined in Equation (10) with the IV matrix $\widehat{Z}=$ $\left[\iota_{n}, X, \widehat{\bar{X}}, \widehat{G} \iota_{n}, \widehat{G} X, \widehat{G} \widehat{\bar{X}}\right]$; and (c) "GMM", the GMM estimator defined in Equation (11).

To obtain the predicted adjacency matrix $\widehat{G}$, we run a logistic regression to predict link formation probabilities based on Equation (9). The estimation results are reported in Table 2. We find strong evidence of homophily, i.e., students are more likely to be friends if they are in the same grade, of the same age, gender and race. The McFadden's pseudo- $R^{2}$ of the logistic regression is 0.15 , suggesting that the above dyadic characteristics are not very informative in predicting friendship formation and, thus, the IV matrix $\widehat{Z}$ constructed using the predicted adjacency matrix $\widehat{G}$ is likely to be weak. In this case, the "GMM" estimator is more reliable than the "2SLS-2" estimator as shown in the Monte Carlo experiments reported in Appendix A.

\section{[Insert Table 2 here]}

The estimation results for Equation (6) are reported in Table 3. For the "2SLS1" estimator, the p-value of the over-identifying restrictions (OIR) test is relatively small, casting doubt on the "conventional" IVs. On the other hand, for the "2SLS2" and "GMM" estimators, the p-values of the OIR test are much higher, lending us confidence in the estimation results. For the social-interaction coefficient $\lambda$, the "2SLS-2" and "GMM" yield similar estimates, while the more efficient "GMM" estimator produces a much smaller standard error. Thus, the "GMM" estimate of $\lambda$ is statistically significant. The uniqueness of our data where both respondents and friends are interviewed allows us to control for friends' characteristics, thus disentangling the endogenous social-interaction effect from exogenous contextual effects (Manski, 1993; see Table 1 for a complete list of contextual variables). Furthermore, the "GMM" estimate of $\lambda$ satisfies the condition $|\lambda|<1 / \rho(G)$ for the existence of 
a unique Nash equilibrium of the underlying network game. Table 4 collects the estimation results with alternative sets of regressors. The last column of this table is identical to the last column of Table 3. The "GMM" estimates of $\lambda$ do not vary much across columns, showing the robustness against potential model misspecifications.

[Insert Tables 3 and 4 here]

\section{$5 \quad$ Key Player Analysis}

The key player is the agent whose removal from the network reduces aggregate activity level the most in equilibrium (Ballester et al., 2006). Under the invariant network assumption (i.e., the rest of the network remains the same after an agent is removed), the key player is the agent with the highest generalized intercentrality measure defined in Section 2.2. With the GMM estimates reported in Table 3, the generalized intercentrality measure can be calculated for each agent and the key player can thus be easily identified.

The invariant network assumption is reasonable in the short run. In the long run, it can be justified if network links are pairwise independent, because in this case the removal of a node and its associated links would not affect the other links in the network. To test the pairwise independence of network links and develop a framework for the key player analysis without imposing the invariant network assumption, we consider a more general network formation model. In the same spirit as Mele (2017), we assume agent $i$ obtain utility from network $g$ given by

$$
\mathcal{U}_{i}(g)=\sum_{j=1}^{n} g_{i j}\left(\delta_{0}+W_{i j} \delta_{1}+\delta_{2} g_{j i}+\delta_{3} \sum_{k \neq i, j} g_{i k}+\delta_{4} \sum_{k \neq i, j} g_{j k} g_{k i}+\eta_{i}+\eta_{j}\right)
$$

As in Section $3, W_{i j}$ is a vector of exogenous variables capturing the similarity between agents $i$ and $j$, with the coefficient vector $\delta_{1}$ representing the homophily or heterophily 
effect. When agent $i$ nominates agent $j$ as a friend, she receives a utility $\delta_{2}$ if $j$ also nominates her as a friend (i.e., $g_{j i}=1$ ). Therefore, $\delta_{2}$ represents the reciprocity effect. We assume the marginal (dis)utility of adding a new link increases with the number of existing links of agent $i$ given by $\sum_{k \neq i, j} g_{i k}$, with the coefficient $\delta_{3}$ representing the congestion effect. Finally, when agent $i$ creates a link to agent $j$, she receives a utility $\delta_{4}$ if $j$ is indirectly linked to $i$ through $k$ (i.e., $g_{j k} g_{k i}=1$ ). Therefore, the coefficient $\delta_{4}$ represents the cyclic-triangle effect. The coefficients $\delta_{3}$ and $\delta_{4}$ reflect the externality of the links between agents $i$ and $j$ on other links in the network. If $\delta_{3} \neq 0$ or $\delta_{4} \neq 0$, then the network links are not pairwise independent.

The utility function defined in Equation (12) admits a potential function in the form of Equation (8) with

$$
m_{i j}(g)=\delta_{2} g_{j i} / 2+\delta_{3} \sum_{k \neq i, j} g_{i k}+\delta_{4} \sum_{k \neq i, j} g_{j k} g_{k i} / 3
$$

As

$$
\mathcal{U}_{i}\left(g_{i j}=1, g_{-i j}\right)-\mathcal{U}_{i}\left(g_{i j}=0, g_{-i j}\right)=\mathcal{Q}\left(g_{i j}=1, g_{-i j}\right)-\mathcal{Q}\left(g_{i j}=0, g_{-i j}\right),
$$

for all $i$ and $j$, where $g_{-i j}$ denote the network including all links in $g$ but $g_{i j}$, the potential function aggregates the individual incentives to deviate from the status quo and, thus, greatly simplifies the equilibrium analysis.

The network formation process follows stochastic best-response dynamics as in Mele (2017). We assume time is discrete and, in time period $t$, a random chosen agent $i$ meets agent $j$ with probability $0<\rho<1$ (see Mele, 2017, for detailed discussion on the meeting technology). Agent $i$ creates a link to agent $j$, i.e., $g_{i j}^{t}=1$, 


$$
\mathcal{U}_{i}\left(g_{i j}^{t}=1, g_{-i j}^{t-1}\right)+\epsilon_{i j, 1}^{t} \geq \mathcal{U}_{i}\left(g_{i j}^{t}=0, g_{-i j}^{t-1}\right)+\epsilon_{i j, 0}^{t}
$$

where $g^{t}$ denotes the realization of the network in period $t$, and $\epsilon_{i j, 1}^{t}$ and $\epsilon_{i j, 0}^{t}$ follow the type 1 extreme value distribution. As shown in Mele (2017), the network formation process is a Markov chain, and converges to a unique stationary distribution given by the Gibbs measure

$$
\pi(g \mid \delta, \eta)=c(\delta, \eta)^{-1} \exp [\mathcal{Q}(g \mid \delta, \eta)]
$$

where $c(\delta, \eta)=\sum_{g \in \mathcal{G}_{n}} \exp [\mathcal{Q}(g \mid \delta, \eta)]$, with $\delta=\left(\delta_{0}, \delta_{1}^{\prime}, \delta_{2}, \delta_{3}, \delta_{4}\right)^{\prime}$ and $\eta=\left(\eta_{1}, \cdots, \eta_{n}\right)^{\prime}$.

Given an observation on the network $g$ from the stationary distribution defined in Equation (13), we can estimate the model parameters based on the maximum likelihood principle. However, the frequentist maximum likelihood method is impractical due to the computational difficulty in evaluating the normalizing constant $c(\delta, \eta)$ in Equation (13), and Bayesian methods, including the Markov Chain Monte Carlo (MCMC) simulation-based Bayesian method using the Metropolis-Hastings (MH) algorithm (Chib and Greenberg, 1995), would encounter the same problem because the normalizing constant $c(\delta, \eta)$ appears in the posterior distribution of model parameters and the acceptance probability of the $\mathrm{MH}$ algorithm. To overcome this computational difficulty, we adopt the DMH algorithm (Liang, 2010; Mele, 2017) to simulate the posterior distribution of model parameters. In particular, we treat unobserved individual heterogeneity terms $\eta_{i}$ 's as unknown parameters and adopt the Bayesian data augmentation approach to sample $\delta$ together with $\eta$ from the joint posterior distribution. The implementation details of the DMH algorithm are described in Appendix B.

We run the DMH algorithm for 50,000 iterations and drop the first 10,000 draws for burn-in and keep every 20th of the remaining draws to conduct the posterior analysis, i.e., to compute the posterior mean (as a point estimate) and posterior 
variance for each parameter. The estimation results are reported in Table 5. Similar to the simple logistic regression estimated in Section 4.2, we find strong evidence of homophily effects, though the magnitude of these effects are smaller than those reported in Table 2. Furthermore, agent $i$ is more likely to form a link to agent $j$, if $j$ has a direct link to $i$ (i.e., the reciprocity effect $\delta_{2}$ ), or if $j$ has an indirect link to $i$ through agent $k$ (i.e., the cyclic-triangle effect $\delta_{4}$ ). Agent $i$ is less likely to form a link to agent $j$, if $i$ has many existing links (i.e., the congestion effect $\delta_{3}$ ). As the estimates of $\delta_{3}$ and $\delta_{4}$ are statistically significant, the network links are not pairwise independent in our data.

\section{[Insert Table 5 here]}

To evaluate the goodness-of-fit of the general network formation model, we generate 1000 networks from the Gibbs measure defined in Equation (13) with the estimates reported in Table 5. The model's goodness-of-fit is examined by comparing the 1000 generated networks with the observed network in terms of three network statistics: the outdegree (the number of links from a node), the indegree (the number of links to a node), and the number of edge-wise shared partners (the number of shared partners of two connected nodes). Figure 2 shows the distributions of these three network statistics of the observed network (in solid lines), the medians and the $95 \%$ confidence intervals of these three network statistics of the 1000 generated networks (in dashed lines). From Figure 2, we find that the generated networks and the observed network display similar distributions over these three network statistics. This shows that our estimated model is able to capture the main features of the underlying network generating process.

[Insert Figure 2 here] 
To conduct the key player analysis without the invariant network assumption, we remove the agents from the network one at a time and simulate the rewiring of the network links among the remaining $n-1$ agent according to the following algorithm with the estimates reported in Table 5.

Algorithm 1 (Network Formation Simulations) Given $\delta$ and $\eta$, at each iteration:

Step 1 Draw $\widetilde{g}$ from a symmetric proposal distribution $q_{g}(\widetilde{g} \mid g)$, where $g$ denotes the network at the current iteration.

Step 2 Accept $\widetilde{g}$ according to the acceptance probability

$$
\alpha_{g}=\min \left\{1, \frac{\exp [\mathcal{Q}(\widetilde{g} \mid \delta, \eta)]}{\exp [\mathcal{Q}(g \mid \delta, \eta)]}\right\}
$$

As shown in Mele (2017), Algorithm 1 generates a Markov chain of networks whose unique invariant distribution is characterized by Equation (13). For each removed student, we run Algorithm 1 for 10,000 iterations, and use the network generated by the last iteration and the GMM estimates reported in Table 3 to calculate the reduction in total delinquency level given by

$$
\left\|\widehat{y}^{*}(g)\right\|_{1}-\left\|\widehat{y}^{*}\left(g_{-i}\right)\right\|_{1}
$$

where $\widehat{y}^{*}(g)=\left(I_{n}-\widehat{\lambda} G\right)^{-1}\left(\widehat{\beta}_{0} \iota_{n}+X \widehat{\beta}_{1}+\bar{X} \widehat{\beta}_{2}\right)$. We then repeat this procedure 1000 times and determine the key player rankings based on the average reduction in total delinquency level. The main findings of the key player analysis are summarized as follows. 
- Based on the general network formation model, we find that removing the key player reduces the total delinquency level by $7.41 \%$ while removing a random agent reduces the total delinquency level by $1.30 \%$ on average. Therefore, the key-player-targeted policy is more effective in reducing delinquent activities as it takes full advantage of the social interaction effect.

- The key player rankings with and without the invariant network assumption are highly correlated, with Spearman's rank correlation coefficient being 0.9219. Therefore, at least in this application, the generalized intercentrality measure (Ballester and Zenou, 2014) is a good approximation of the key player ranking without the invariant network assumption.

- The key player rankings are moderately correlated with the conventional network centrality measures. The Spearman's rank correlation coefficients are reported in Table 6. As expected, the key player ranking depends on the position of an agent in the network. However, using the network centrality measures alone cannot correctly identify the key player.

- The key player rankings are not much correlated with the delinquent activity involvement, with Spearman's rank correlation coefficient being 0.0358. Based on the general network formation model, we find that removing the most active agent in delinquent activities reduces the total delinquency level by $1.48 \%$. Hence, the most active agent in delinquent activities is not necessary the one whose removal leads to the largest reduction in total delinquency level.

- Finally, to see how the key player ranking is affected by inconsistent estimates of Equation (6), we obtain a key player ranking based on the generalized intercentrality measure evaluated at the 2SLS-1 estimates reported in Table 3. We 
find that the key player rankings with 2SLS-1 and GMM estimates are moderately correlated, with Spearman's rank correlation coefficient being 0.3806. We also find that removing the key player based on the 2SLS-1 estimates only reduces the total delinquency level by $4.36 \%$, and hence is much less effective than removing the key player based on the GMM estimates.

[Insert Table 6 here]

\section{Conclusion}

This paper presents a methodology for empirically identifying key players in networks. This methodology has great scope for practical implementation given the increasing availability of network data in various contexts, including financial networks (Battiston et al., 2012; Cohen-Cole et al., 2014) and political networks (Battaglini and Patacchini, 2018; Battaglini et al., 2019). Compared to a policy that targets the most active agent in the network, the key-player-targeted policy is more effective in reducing the aggregate activity level as it takes full advantage of social interaction effects. In addition, targeting the key player has an advantage over targeting the most active agent in that it is harder for the key player to hide her identity than the most active agent, as the latter only needs to temporarily reduce her activity level in the period of policy intervention (that is, temporarily stop being delinquent when there is a crackdown going on). Given the aforementioned benefits of the key-player-targeted policy, it makes sense to consider such a policy if its benefits outweigh the cost of data collecting when network data is not readily available.

Some extensions of the current framework are possible. First, although we use one network to showcase the key player analysis, the proposed methodology can be readily extended to analyze multiple networks. Second, in the empirical illustration, we focus on petty delinquent activities. If one is interested in identifying the key 
player in more serious offenses that a large fraction of the students do not participate in, then the endogenous participation problem needs to be taken into account when estimating social-interaction effects and conducting the key player analysis. We leave this extension for future research.

\section{References}

Ballester, C., Calvó-Armengol, A. and Zenou, Y. (2006). Who's who in networks. Wanted: the key player, Econometrica 74: 1403-1417.

Ballester, C. and Zenou, Y. (2014). Key player policies when contextual effects matter, Journal of Mathematical Sociology 38: 233-248.

Battaglini, M., Leone Sciabolazza, V. and Patacchini, E. (2019). Effectiveness of connected legislators. Forthcoming in American Journal of Political Science.

Battaglini, M. and Patacchini, E. (2018). Influencing connected legislators, Journal of Political Economy 126: 2277-2322.

Battiston, S., Puliga, M., Kaushik, R., Tasca, P. and Caldarelli, G. (2012). DebtRank: Too central to fail? financial networks, the fed and systemic risk, Scientific Reports 2: 1-6.

Becker, G. (1968). Crime and punishment: An economic approach, Journal of Political Economy 76: 169-217.

Bramoullé, Y., Djebbari, H. and Fortin, B. (2009). Identification of peer effects through social networks, Journal of Econometrics 150: 41-55.

Calvó-Armengol, A., Patacchini, E. and Zenou, Y. (2009). Peer effects and social networks in education, The Review of Economic Studies 76: 1239-1267. 
Chib, S. and Greenberg, E. (1995). Understanding the Metropolis-Hastings algorithm, The American Statistician 49: 327-335.

Cohen-Cole, E., Kirilenko, A. and Patacchini, E. (2014). Trading networks and liquidity provision, Journal of Financial Economics 113: 235-251.

de Paula, A. (2017). Econometrics of network models, in B. Honore, A. Pakes, M. Piazzesi and L. Samuelson (eds), Advances in Economics and Econometrics: Theory and Applications: Eleventh World Congress, Vol. 1, Cambridge University Press, pp. 268-323.

Kelejian, H. H. and Piras, G. (2014). Estimation of spatial models with endogenous weighting matrices, and an application to a demand model for cigarettes, Regional Science and Urban Economics 46: 140-149.

Kelejian, H. H. and Prucha, I. R. (1998). A generalized spatial two-stage least squares procedure for estimating a spatial autoregressive model with autoregressive disturbance, Journal of Real Estate Finance and Economics 17: 99-121.

Kelejian, H. H. and Prucha, I. R. (2010). Specification and estimation of spatial autoregressive models with autoregressive and heteroskedastic disturbances, Journal of Econometrics 157: 53-67.

Lee, L. F. (2007). GMM and 2SLS estimation of mixed regressive, spatial autoregressive models, Journal of Econometrics 137: 489-514.

Liang, F. (2010). A double Metropolis-Hastings sampler for spatial models with intractable normalizing constants, Journal of Statistical Computation and Simulation 80: 1007-1022. 
Lin, X. and Lee, L. F. (2010). GMM estimation of spatial autoregressive models with unknown heteroskedasticity, Journal of Econometrics 157: 34-52.

Liu, X. and Lee, L. F. (2010). GMM estimation of social interaction models with centrality, Journal of Econometrics 159: 99-115.

Manski, C. F. (1993). Identification of endogenous social effects: the reflection problem, The Review of Economic Studies 60: 531-542.

Mele, A. (2017). A structural model of dense network formation, Econometrica 85: 825-850.

Monderer, D. and Shapley, L. (1996). Potential games, Games and Economic Behavior 14: 124-143.

Zenou, Y. (2016). Key players, in Y. Bramoullé, A. Galeotti and B. Rogers (eds), Oxford Handbook on the Economics of Networks, Oxford University Press, pp. 133. 
Figure 1: Friendship Nomination Network

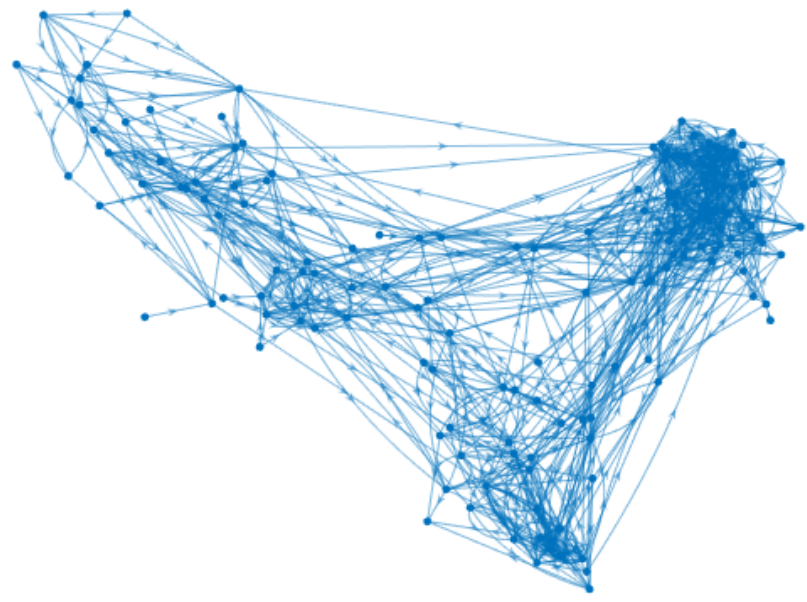


Figure 2: Goodness of Fit of the General Network Formation Model
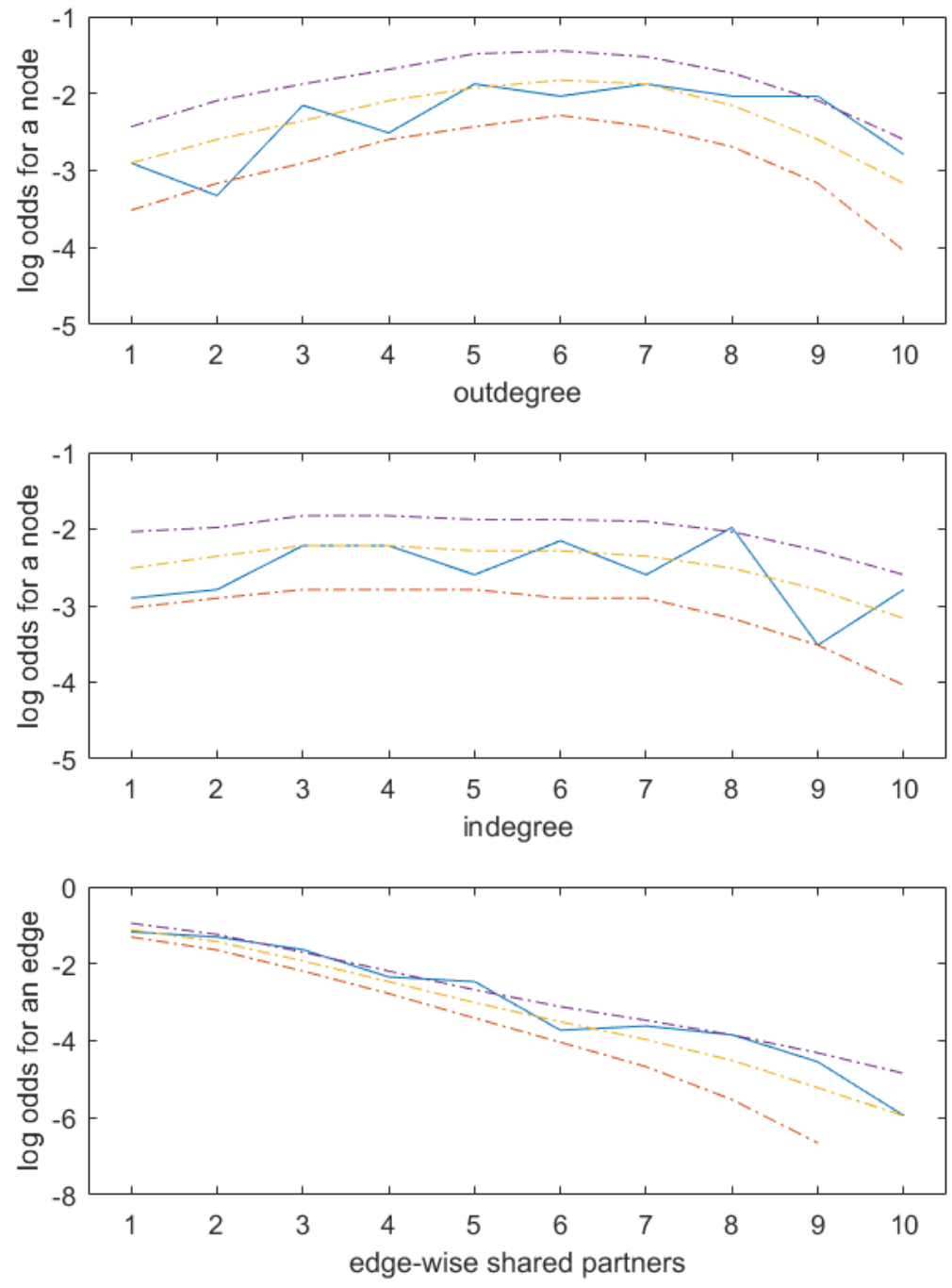

Notes: The distributions of the observed network are in solid lines, while the medians and the $95 \%$ confidence intervals of the 1000 generated networks are in dashed lines. 


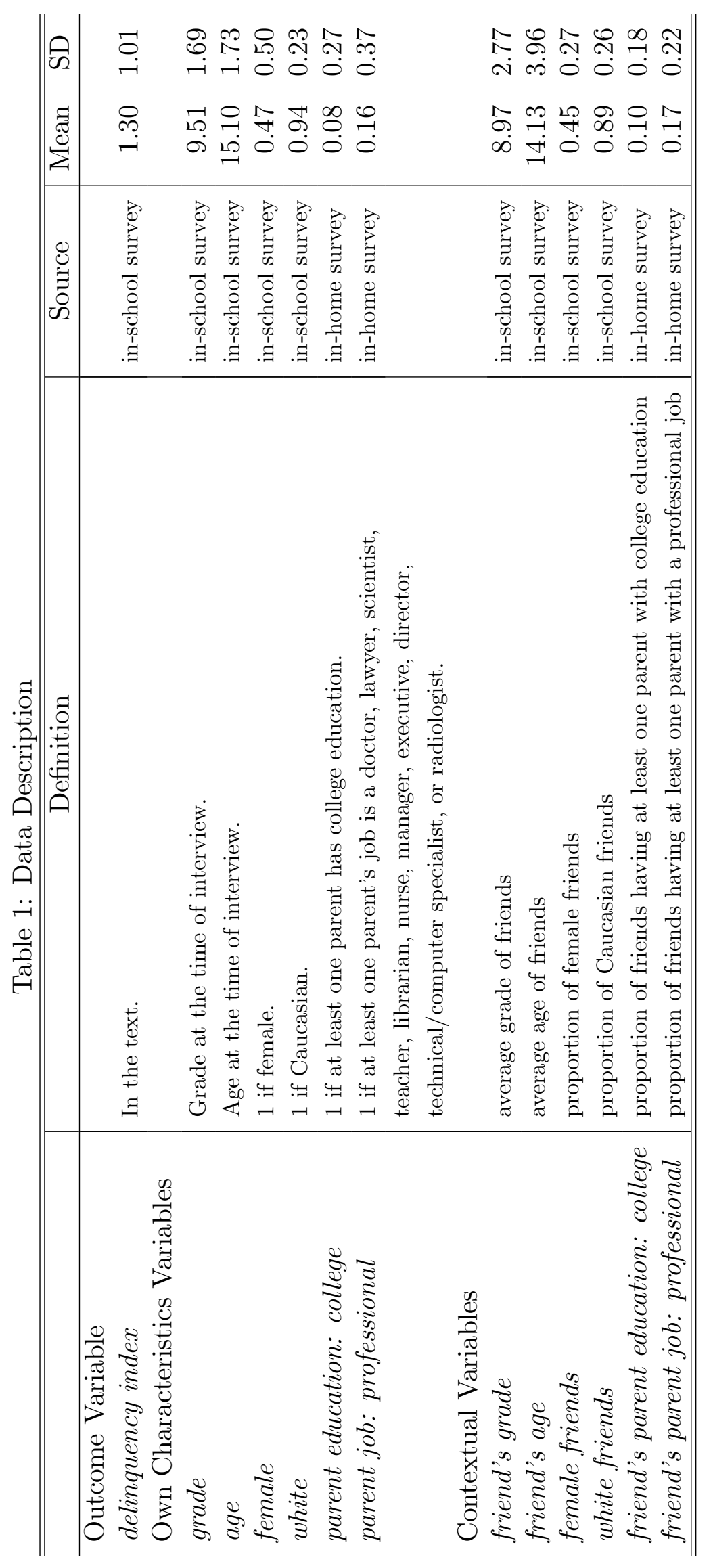


Table 2: Logistic Regression of Link Formation

\begin{tabular}{l|l}
\hline \hline same grade & $2.2602^{* * *}$ \\
& $(0.0755)$ \\
same age & $0.4075^{* * *}$ \\
& $(0.0752)$ \\
same gender & $0.2337^{* * *}$ \\
& $(0.0680)$ \\
same race & $0.2373^{* *}$ \\
& $(0.1204)$ \\
\hline McFadden's pseudo $R^{2}$ & 0.150 \\
\hline \hline
\end{tabular}

Standard errors in parentheses.

Statistical significance: ${ }^{* * *} \mathrm{p}<0.01 ;{ }^{*} \mathrm{p}<0.05 ;{ }^{*} \mathrm{p}<0.1$.

Table 3: 2SLS and GMM Estimation Results

\begin{tabular}{l|ccc}
\hline \hline & 2SLS-1 & 2SLS-2 & GMM \\
\hline$\lambda$ & -0.0269 & 0.0934 & $0.0855^{* *}$ \\
grade & $(0.0262)$ & $(0.1114)$ & $(0.0429)$ \\
& 0.1071 & -0.0253 & 0.0659 \\
age & $(0.1273)$ & $(0.6807)$ & $(0.4211)$ \\
female & 0.1133 & -0.0400 & -0.0179 \\
& $(0.1075)$ & $(0.2638)$ & $(0.1961)$ \\
white & $-0.4303^{* * *}$ & $-1.2868^{* *}$ & $-1.0321^{* *}$ \\
& $(0.1641)$ & $(0.5987)$ & $(0.5244)$ \\
parent education: college & -0.4810 & -0.8562 & -0.7164 \\
& $(0.3158)$ & $(0.6220)$ & $(0.5427)$ \\
parent job: professional & -0.2792 & -0.4626 & -0.4785 \\
& $(0.2288)$ & $(0.5347)$ & $(0.3545)$ \\
contextual variables & -0.0803 & -0.4867 & -0.1684 \\
Cragg-Donald Wald F statistic & $(0.2291)$ & $(0.5694)$ & $(0.4287)$ \\
OIR test p-value & 27.329 & 0.272 & - \\
\hline \hline
\end{tabular}

Heteroskedasticity-robust standard errors in parentheses.

Statistical significance: ${ }^{* * *} \mathrm{p}<0.01 ;{ }^{* *} \mathrm{p}<0.05 ;{ }^{*} \mathrm{p}<0.1$. 
Table 4: GMM Estimation Results with Alternative Model Specifications

\begin{tabular}{l|llll}
\hline \hline$\lambda$ & $0.0771^{* *}$ & $0.0665^{*}$ & $0.0823^{*}$ & $0.0855^{* *}$ \\
grade & $(0.0348)$ & $(0.0347)$ & $(0.0420)$ & $(0.0429)$ \\
& -0.1135 & -0.1208 & 0.3190 & 0.0659 \\
age & $(0.1500)$ & $(0.1491)$ & $(0.7517)$ & $(0.4211)$ \\
& 0.1807 & 0.1949 & -0.0572 & -0.0179 \\
female & $(0.1341)$ & $(0.1321)$ & $(0.2788)$ & $(0.1961)$ \\
& $-0.3997^{* * *}$ & $-0.3863^{* * *}$ & -1.0753 & $-1.0321^{* *}$ \\
white & $(0.1485)$ & $(0.1480)$ & $(0.6549)$ & $(0.5244)$ \\
& -0.4172 & -0.3515 & -0.8429 & -0.7164 \\
parent education: college & $(0.2883)$ & $(0.2759)$ & $(0.5589)$ & $(0.5427)$ \\
& & -0.2590 & & -0.4785 \\
parent job: professional & & $(0.2234)$ & & $(0.3545)$ \\
& & 0.0162 & & -0.1684 \\
& & $(0.1862)$ & & $(0.4287)$ \\
\hline contextual variables & no & no & yes & yes \\
OIR test p-value & 0.277 & 0.253 & 0.972 & 0.867 \\
\hline \hline
\end{tabular}

Heteroskedasticity-robust standard errors in parentheses.

Statistical significance: ${ }^{* * *} \mathrm{p}<0.01 ;{ }^{* *} \mathrm{p}<0.05 ;{ }^{*} \mathrm{p}<0.1$.

Table 5: Estimates of the General Network Formation Model

\begin{tabular}{l|c}
\hline \hline same grade & $1.5420^{* * *}$ \\
same age & $(0.0683)$ \\
& $0.2531^{* * *}$ \\
same gender & $(0.0685)$ \\
& $0.2422^{* * *}$ \\
same race & $(0.0585)$ \\
& 0.1168 \\
the reciprocity effect $\delta_{2}$ & $(0.1700)$ \\
& $2.4764^{* * *}$ \\
the congestion effect $\delta_{3}$ & $(0.0935)$ \\
& $-0.0970^{* * *}$ \\
the cyclic-triangle effect $\delta_{4}$ & $(0.0118)$ \\
& $0.4538^{* * *}$ \\
\hline Unobserved individual heterogeneity & $(0.0446)$ \\
\hline \hline
\end{tabular}

Standard errors in parentheses.

Statistical significance: ${ }^{* * *} \mathrm{p}<0.01 ;{ }^{* *} \mathrm{p}<0.05 ;{ }^{*} \mathrm{p}<0.1$. 
Table 6: Spearman's Rank Correlation Coefficients

\begin{tabular}{l|c}
\hline \hline & key player rankings \\
\hline outdegree centrality & 0.2794 \\
indegree centrality & 0.2850 \\
incloseness centrality & 0.2644 \\
outcloseness centrality & 0.2229 \\
betweenness centrality & 0.3566 \\
\hline \hline
\end{tabular}

